\title{
Desafíos de la Educación en Tiempos de Cambio SOCIAL
}

\author{
Educational Challenges in Times of Social Changes
}

\author{
Emma Salas Neumann \\ Especialista en Orientación Educacional y Vocacional \\ El Vergel 2271, Dpto. 1001 \\ Providencia, Santiago \\ silviasalas@vtr.net
}

Recibido: 17 de noviembre, 2008. Aceptado: 19 de noviembre, 2008

Resumen: Se presenta aquí una reflexión inspirada en las controversias a que está sometida la educación del país en nuestros días. Se plantea la necesidad de reconocer los cambios sociales y culturales y su influencia en la educación, de modo de redefinir el rol de la escuela para atender las necesidades de una sociedad que se renueva constantemente. Esta exige renovadas visiones de la vida y nuevas competencias para afrontar los frecuentes cambios sociales y culturales a los que el individuo estará sometido, en diversos ámbitos, durante su lapso de vida. Se requiere preparar al estudiante para un aprendizaje continuo y una frecuente toma de decisiones con respecto a sí mismo, para lo cual es necesario renovar el ambiente del aula, la relación profesor-alumno y la dinámica interna de la escuela, así como la organización del currículo y sus contenidos.

Palabras clave: educación, cambio, potencialidades, diversidad, democratización.

Abstract: Here is presented a personal reflection on the controversial arguments used in public discussion about education which takes place in the country nowadays. The importance to consider how social change affects education, and highlights the need to change traditional practices in order to meet new needs is emphasized . Schools require the promotion of new visions of life and the developing of other competences to cope with the frequent social and cultural changes part of any individual's life along his/her life in a changing society, to live in a changing society. Schools should prepare students for a continuous learning and a frequent decision making about him/herself throughout all life. To reach those objectives, schools have to renew classroom atmosphere, teacher-pupil relationship, school inner dynamics and curriculum organization and contents.

Keywords: education, change, potentialities, diversity, democratization.

\footnotetext{
*Presentación en Panel sobre Desafíos de la Educación, en Coloquio Los desafíos del sistema escolar chileno, organizado por la Corporación de Graduados y el Departamento de Educación de la Facultad de Ciencias Sociales de la Universidad de Chile, 29 de octubre, 2008.
} 


\section{INTRODUCCIÓN}

Nuestra educación es hoy muy criticada, contrariamente al prestigio de que gozó históricamente. Una posible respuesta es que en el pasado la educación que se ofrecía era adecuada a la sociedad de cambio lento en que estaba inmersa y atendía a una población mucho menos numerosa y también menos diversa culturalmente. Esa era una sociedad con más certezas, más estable. Hoy la sociedad es distinta, su futuro es más incierto y exige a sus integrantes estar preparados para asumir cambios y adquirir nuevas competencias para integrarse y permanecer en esa sociedad compleja y más desafiante para el ser humano.

Sin embargo, la imagen cultural que la sociedad tiene de la educación tiende a ser aún algo tradicional y no se advierte una percepción más certera de la influencia social del Cambio, que implica miradas que representen la esencia de la educación dentro de un contexto histórico distinto. Entonces, el análisis de experiencias educativas anteriores no contribuye a visualizar problemas presentes.

\section{LA EDUCACIÓN Y EL CAMBIO}

El sistema escolar de hoy debe atender a una mayor población, más diversa y socializarla para retos inciertos y ello es, en si mismo, un desafío para la educación. Quizás, esa puede ser una de las razones de que la educación sea víctima de una serie de contradicciones culturales que afectan su finalidad, orientación y contenido, así como el rol del profesor.

Hoy se habla de democratización dela educación, calidad dela educación, igualdad de oportunidades, inclusión, equidad, como características que debe tener el sistema escolar. Todas las nombradas están interrelacionadas y son aspiraciones de antigua data, las que hoy, enriquecidas por los avances del saber y mejor explicitadas -aunque a veces no son reconocidas en los tiempos actuales- siguen respondiendo a características deseables del sistema escolar.

\section{LA DEMOCRATIZACIÓN DE LA EDUCACIÓN, SU SIGNIFICADO HOY}

La democratización de la educación significa, básicamente, que todos los integrantes de una sociedad, reciban una educación que les permita desarrollar sus potencialidades para insertarse en la sociedad a la que pertenecen. Ello implica un acceso amplio al sistema escolar y que éste posea la diversidad necesaria para el desarrollo de una variedad de potencialidades y prácticas de vida. Esto facilitaría la retención de los estudiantes en el sistema escolar 
hasta el logro de los aprendizajes requeridos para así integrarse a la sociedad a que pertenecen, completando los ciclos de estudio inicialmente planeados.

Sin embargo, a través de la democratización de la educación no sólo se aspira a que todos reciban la educación que requieran, sino que también se espera que alcancen la formación de una mentalidad democrática como fundamento de la ciudadanía. Ello implica, por una parte, la existencia de un sistema que, a través del currículo, ofrezca oportunidades de desarrollar una variedad de potencialidades y la posibilidad de que el sujeto pueda explorar sus atributos para tomar decisiones fundamentadamente. Por otra parte, requiere que el alumno sea expuesto, tanto en la escuela como en el aula, a una experiencia de convivencia democrática, lo cual explica la tendencia a considerar una renovada forma de relación profesor-alumno.

Es necesario tener presente la importancia de la atención a la diversidad de la población que acude a las aulas. Y esa diversidad es atendida no sólo por la pertinencia y variedad de currículo, sino también por la variedad metodológica que el docente despliegue. A ello se agrega el clima 0 atmósfera del aula que permita la reacción espontánea de los estudiantes al confrontar su propia experiencia con los nuevos saberes y experiencias que ocurren en el espacio docente, de modo que los alumnos aprendan a participar responsablemente. Es ese el clima psicológico que facilita los aprendizajes más importantes como son los de relacionar hechos, clasificar y priorizar materias e intereses, integrar experiencias y adquirir estrategias para aplicar saberes y muchos otros procesos que constituyen la base del aprender a aprender, tan necesario para vivir en una sociedad que cambia aceleradamente.

Por otra parte, ese clima psicológico del aula al que nos hemos referido es el que proporciona las bases de la formación de una mentalidad democrática, como fundamento de la ciudadanía, al que hemos aludido anteriormente. Es el desafío de lograr las características de aquella persona que escucha, que acepta la diversidad de opiniones y que es capaz de llegar a ciertos consensos en aras de una tarea conjunta superior.

\section{OTRO CONCEPTO DE DISCIPLINA ESCOLAR}

Muy relacionado con el tema anterior, el clima del aula, debe considerarse el concepto de disciplina escolar que la escuela aplique. Aquella más tradicional, que se basaba en una relación algo lejana con el profesor, ha quedado atrás; éste representaba una especie de autoridad sin contrapeso y poseedor de los saberes que presentaba y explicaba a los estudiantes, quienes debían escuchar silenciosos. Hoy contamos con estudios sobre dinámica de 
grupos que han permitido valorar el curso como un grupo y no como una suma de individualidades, como lo fue en el pasado. Esa valoración significa que el curso, principalmente por la acción del profesor-jefe, debe convertirse, a través de la interacción de sus miembros, en un grupo integrado que constituya un espacio privilegiado para aprendizajes sociales y buena convivencia en el cual los estudiantes vivan los valores en lo cotidiano (objetivos transversales) y conozcan y reconozcan las actitudes que los expresan.

\section{ALGUNOS DESAFÍOS ESPECÍFICOS DE LA ACELERACIÓN DEL CAMBIO}

\section{El mundo del trabajo y el cambio}

El mundo del trabajo al cual, en una u otra forma, la gran mayoría de la juventud en formación se incorporará, tiene características crecientemente distintas a las de antaño, y que seguramente se intensificarán en el futuro. No importa el nivel de la actividad laboral que se realice, ésta estará sometida a modificaciones que obligarán al individuo a incorporar nuevos saberes 0 formas de acción en su actividad laboral, descubrir un nuevo campo de actividad 0 aplicación de sus saberes, es decir, imaginar respuestas al cambio, aprender constantemente y conservar la capacidad de adaptarse a nuevas modalidades de trabajo. Lo cierto es que el campo laboral se ha tornado cada vez más incierto y requiere flexibilidad psicológica y social para aceptar los cambios. Sin embargo, la información vocacional que aún se ofrece en la escuela tiende a ser tradicional y para un mundo laboral bastante estable.

\section{Percepción del cambio por parte del individuo}

Los cambios no ocurren sólo en el mundo laboral, sino también trascienden la vida en comunidad. El individuo que vive en el siglo XXI percibirá que el cambio se acelera, al afectarle varias veces en su vida y en lapsos cada vez más frecuentes, lo cual puede provocar en muchos de ellos angustia y sensación de inestabilidad. Lo sentimos, por ejemplo, cuando debemos romper hábitos de vida para realizar lo mismo de otra manera; cuando lo que sabíamos de una materia determinada es hoy distinto 0 al menos insuficiente; cuando las situaciones de la vida cotidiana deben atenderse de modo distinto a lo que creíamos adecuado; cuando es necesario aceptar conductas de convivencia que antes no nos parecían recomendables; cuando en nuestra actividad de trabajo se nos presentan situaciones nuevas que no podemos resolver como antes. 


\section{Distancia cultural generacional}

La aceleración del cambio genera, frecuentemente, una distancia cultural entre generaciones que no sólo afecta la relación padres-hijos, sino igualmente la distancia cultural profesor-alumno. Seguramente, esa distinta percepción de actitudes y conductas entre generaciones es responsable de algunos de los conflictos que asombran hoy a los adultos más tradicionales.

En estas circunstancias, si no se reconocen estas diferencias y si en el caso de la comunidad escolar se aplica un concepto tradicional de disciplina escolar, en la cual no hay comunicación y si hay diálogo, éste es escaso, la distancia cultural se agudiza e interrumpe la comunicación.

\section{Condiciones de trabajo en la escuela}

Los integrantes más importantes de la vida cotidiana de una escuela son los profesores y estudiantes, a esto se agrega el entorno escolar, las condiciones de trabajo y la existencia de ayudas pedagógicas, que incluyen hoy los computadores e Internet, instrumentos significativos que amplían la mirada a otros mundos.

Sin embargo, lo más importante sigue siendo, quizás, el clima participativo y de libertad responsable que debe reinar en la comunidad escolar, la relación profesor-alumno y las condiciones para que ésta sea lo más fructífera posible.

Los grupos-curso demasiado numerosos no contribuyen a una mejor y más cercana relación entre profesores y estudiantes y a un clima favorable a la participación. Hoy que el grupo familiar ha perdido las características de estabilidad y seguridad para algunos niños y jóvenes escolares, esta relación más cercana, en especial con el profesor-jefe, puede constituirse en un Ancla para aquellos estudiantes que carecen de apoyo y orientación, pero para que ello ocurra los grupos deben ser más reducidos.

\section{Los profesores y el cambio}

Uno de los temas que más preocupa es el de los profesores a quienes se responsabiliza prioritariamente de los resultados de las pruebas que miden los aprendizajes, aunque éstos son preferentemente del área intelectual. Se cuestiona su formación inicial, por requerir seguramente reorientaciones y actualizaciones, pero creemos que ello es absolutamente insuficiente si consideramos la aceleración del Cambio. 
Estimo que la formación inicial debe atenuar las consecuencias del Cambio tecnológico, social y cultural, lo que lleva a dos consideraciones principales:

1. Generar una comprensión a cabalidad de la influencia de las formas de vida y del entorno en que han crecido y viven los estudiantes en comparación con lo que espera la cultura escolar, de modo de comprender mejor sus conductas y actitudes. Esto es especialmente importante cuando estamos defendiendo la democratización de la educación y la igualdad de oportunidades, además de la inclusión. Ello perfecciona la comunicación entre estudiantes y profesores y permite llegar a un terreno común de entendimiento que es la base de aprendizajes sociales y de acercamiento para una más fructífera relación profesor-alumno.

2. Considerar los saberes generales y específicos que deben mantener los profesores a través de su carrera. Es decir, proporcionar oportunidades de mantener al día a los docentes acerca de las nuevas tendencias del saber que deben compartir con los estudiantes.

Hasta ahora, frecuentemente se han ofrecido cursos de perfeccionamiento de diversa efectividad. No obstante, me parece importante lo que podría llamarse perfeccionamiento continuo en servicio, asociado a las tareas cotidianas del profesor, lo que no implicaría restarse a cursos de perfeccionamiento actuales.

Sin Embargo, para lo que hemos denominado "perfeccionamiento en servicio", se requieren algunas modificaciones en las condiciones de trabajo de los profesores. En primer término deberían existir horas dedicadas específicamente para que funcionen grupos de estudio dentro de la escuela que traten temas relativos a una docencia más eficiente. Estos grupos de estudio estarían organizados para un trabajo sistemático bajo la tuición de la persona 0 personas encargadas de la Unidad Técnico Pedagógica (UTP) existentes en los establecimientos educacionales; estos profesores deben ser personas preparadas para ejercer ese liderazgo. Se trata de una reflexión conjunta de pares sobre situaciones cotidianas que ocurren en la escuela y que muestran problemas de ocurrencia frecuente. Preguntarse, por ejemplo, cuáles son los objetivos y contenidos prioritarios de los extensos programas de estudio más adecuados para que los estudiantes, más que contenidos, adquieran 0 aprendan a pensar, relacionar, conocer, buscar, clasificar, aplicar percepciones e informaciones y otros procesos que les permitirán aprender a tomar decisiones en distintos momentos y ámbitos de su vida. ¿Cómo proporcionar un ambiente grato y participativo que permita la expresión genuina de los estudiantes? En suma, cómo formar personas, que dentro de 
sus personales atributos compartan actitudes para convivir en una sociedad plural y democrática.

Los profesores también requieren una disciplina profesional, pero al igual que aquella que se aplica a los alumnos, debe darse en un contexto de liderazgo democrático, es decir, escuchar a los subalternos, a esto se agregarían horas destinadas a perfeccionamiento interno. Si queremos reencantar la docencia para que las personas disfruten su trabajo y se sientan valoradas en su actividad laboral para obtener resultados adecuados, es necesario poner atención a las rutinas escolares y a las condiciones de trabajo.

El descontento que se percibe hoy con respecto a la educación nacional amerita no sólo la aclaración de ciertos conceptos que consideren el nuevo contexto histórico, sino también una mirada global al rol de la educación en una sociedad que cambia y renueva su cultura aceleradamente y obliga al individuo a tomar decisiones sobre sí mismo con frecuencia.

Priorizar en esa mirada global diversos aspectos relacionados con la organización, financiamiento y dinámica interna de la escuela de modo de identificar ripios dejados por rutinas de larga data que dificultan las modificaciones que requiere la educación para una sociedad afectada por el Cambio. 